\title{
An immunological and histoimmunological study of gastric sulphoglycoproteins in healthy and aspirin-treated dogs
}

\author{
I. P. T. HÄKKINEN, R. JOHANSSON, AND M. PANTIO \\ From the Departments of Medical Microbiology and Zoology, University of Turku, Turku, Finland
}

The macroscopic lesions in gastric mucosal lining caused by orally ingested aspirin are obvious both in experimental animals and in man (Grossman, Matsumoto, and Lichter, 1961; Roth, ValdesDapena, Pieses, and Buchman, 1963). A tendency to multiple small superficial erosions and bleedings is well known. The lesions are readily caused by the oral application of aspirin, but at least in experimental conditions similar gastric lesions have also been noted after parenteral administration, thus making valid the assumptions about the existence of a more specific effect than 'local burning'.

The inhibitory effect of aspirin on oxidative phosphorylation in cell has been investigated (Whitehouse, 1964). Salicylates inhibit the synthesis of connective tissue polysaccharides in fibroblasts (Whitehouse and Boström, 1962). The superficial gastric mucous cells synthesize glycoproteins which behave in chemical analysis very much like the connective tissue polysaccharides and contain as much as 80 to $90 \%$ of sugar components. The pathways of their biosynthesis are presumably closely related (Robbins, Bray, Dankert, and Wright, 1967).

A significant and important component of glycoproteins in gastric superficial epithelium is the sulphated glycoprotein. The isolation and chemical analysis of gastric sulphoglycoproteins has recently been performed (Häkkinen, Hartiala, and Terho, 1965; Häkkinen, 1967), and the preservation of these macromolecules as intact as possible in the isolation procedure for immunological identification and for analysis with physico-chemical methods has been the aim in successive studies (Häkkinen, 1966, 1967). A highly sensitive method for localizing human gastric sulphoglycoproteins in tissue sections is the indirect immunofluorescence technique (Häkkinen, Grönroos, and Kunnas, 1967). In the present work the new immunological techniques mentioned above have been applied in an attempt to identify and localize sulphoglycoproteins in the gastric mucosa of the dog and to study the possible effect of aspirin upon them.

\section{MATERIALS AND METHODS}

The material consisted of 12 adult mongrel dogs of both sexes. Aspirin tablets were fed orally in one daily dose of $70 \mathrm{mg} / \mathrm{kg}$ body weight (to four dogs) or $140 \mathrm{mg} / \mathrm{kg}$ body weight (to four dogs) during a period of three and a half months. Four dogs served as controls. Mixed food and water was freely given over the whole period. The dogs were killed with an intravenous injection of 5 to $10 \mathrm{ml}$ of nembutal. The stomachs were removed immediately, opened, roughly cleaned avoiding water, quickly frozen, and stored at $-20^{\circ} \mathrm{C}$ as plates in tight plastic bags until used.

Five pieces from each stomach were cut out with a stance at even distances using a common model from the distal antrum to the upper part of the fundus. All the pieces were taken from macroscopically healthy tissue. They were fixed in butanol and embedded in paraffin and sections of $5 \mu$ were cut for histochemical and immunofluorescence studies.

Specific rabbit immunosera prepared against canine gastric sulphoglycoproteins were used in the indirect immunofluorescence technique (rabbit immunoserumantirabbit sheep $\gamma$-globulin isothiocyanate fluorescein conjugate) as described earlier (Häkkinen et al, 1967).

The histochemical stains were alcian blue at $p \mathrm{H} 1$ and alcian blue at $p \mathrm{H} 1$ with PAS (Spicer and Sun, 1967). To avoid the loss of readily water-soluble sulphoglycoproteins the de-paraffined sections were transferred from $80 \%$ ethanol directly to the staining solutions.

Sulphoglycoproteins were isolated from the gastric juice of three dogs fed with aspirin for two and a half months and of four controls. Before collecting the gastric juice the dogs had been fasted for two days. For the collection procedure, $300 \mathrm{ml}$ of saline was aspirated through an orally introduced stomach tube and mixed with gastric contents, which then were withdrawn through a record syringe.

The samples from the aspirin dogs were pooled as well as those from control dogs. The further treatment and isolation technique have already been described (Häkkinen, 1966, 1967). The acidity of the sample was adjusted with $0.1 \mathrm{~N}$ bicarbonate solution to $p \mathrm{H} 6$ to 6.5 , then $1 \mathrm{vol}$ of $1 \%$ cetylpyridinium chloride (CPC) was added to $4 \mathrm{vol}$ of the sample and allowed to stand for half an hour. The precipitate thus formed was centrifuged, the supernatant discharged, and the precipitate dissolved to about $2 \mathrm{ml}$ 712 
of $2 \mathrm{~N} \mathrm{MgCl}$. The sulphoglycoproteins were reprecipitated from the $\mathrm{MgCl}_{2}$-solution with $4 \mathrm{vol}$ of $96 \%$ ethanol and dried with ether. Dry material was dissolved in saline, centrifuged, and only the clear supernatant was accepted for immunization, double immunodiffusion, or polyacrylamide electrophoresis.

The immunization of rabbits was performed with complete Freund's adjuvant using intradermal injections to five points near the extremities and middle back. A second booster was given after one month. Blood was collected after 10 more days.

In double immunodiffusion the same microplate modification of Häkkinen (1967) was used.

Electrophoresis using polyacrylamide discs was performed in circumstances adapted for estimating serum proteins either in the sparse gel alone or together with drive gel as support (Davis, 1964). The discs were stained with alcian blue in $7 \%$ acetic acid.

\section{RESULTS}

Six of the eight dogs belonging to the aspirin group developed small multiple erosions and bleeding in the gastric mucosa. No apparent difference could be observed between the subgroups (which received $70 \mathrm{mg}$ or $140 \mathrm{mg}$ of aspirin per $\mathrm{kg}$ of body weight respectively). The two dogs without macroscopical lesions also belonged to separate groups. The erosions showed a tendency to localize in the antrum or intermediate zone. The stomachs of the control animals were macroscopically intact.

GASTRIC SULPHOGLYCOPROTEINS One of the sulphoglycoprotein components in the gastric juice of the control group was absent in the gastric juice of aspirin dogs as shown by the double immunodiffusion study (Fig. 1). When the electrophoretic patterns

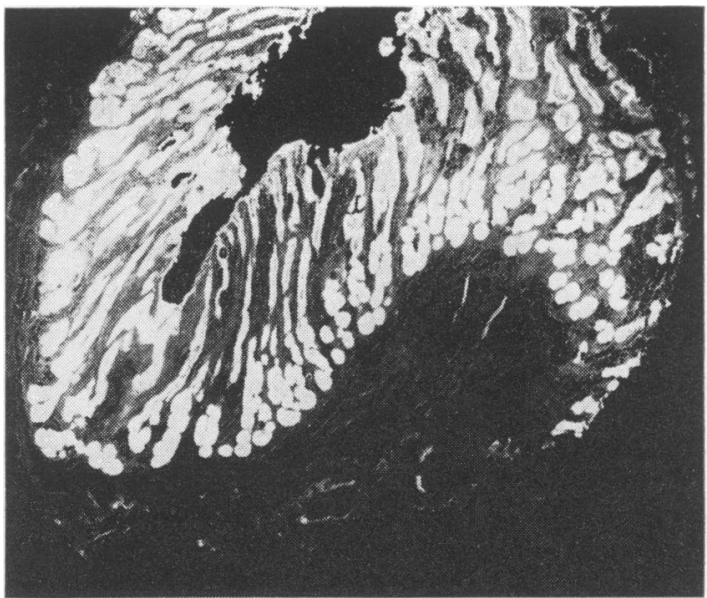

FIG. 2. Photomicrograph in ultraviolet light of a section from the antrum of a stomach in control group stained with rabbit antiserum prepared against gastric sulphoglycoproteins of aspirin group and stained with antirabbit sheep $\gamma$-globulin isothiocyanate fuorescein conjugate. Both superficial and deep glands are specifically stained.

of sulphoglycoproteins from control and aspirin groups are compared, the aspirin group shows an increased polydispersion which appears as division into a greater number of bands in tight molecular sieve support. However, this phenomenon disappears when the support is more porous.

The typical results of the immunofluorescence technique are presented in Figures 2 to 9. The intensity of the specific fluorescence in the mucosa of all control dogs is high compared with that seen in aspirin dogs. There was no significant variation
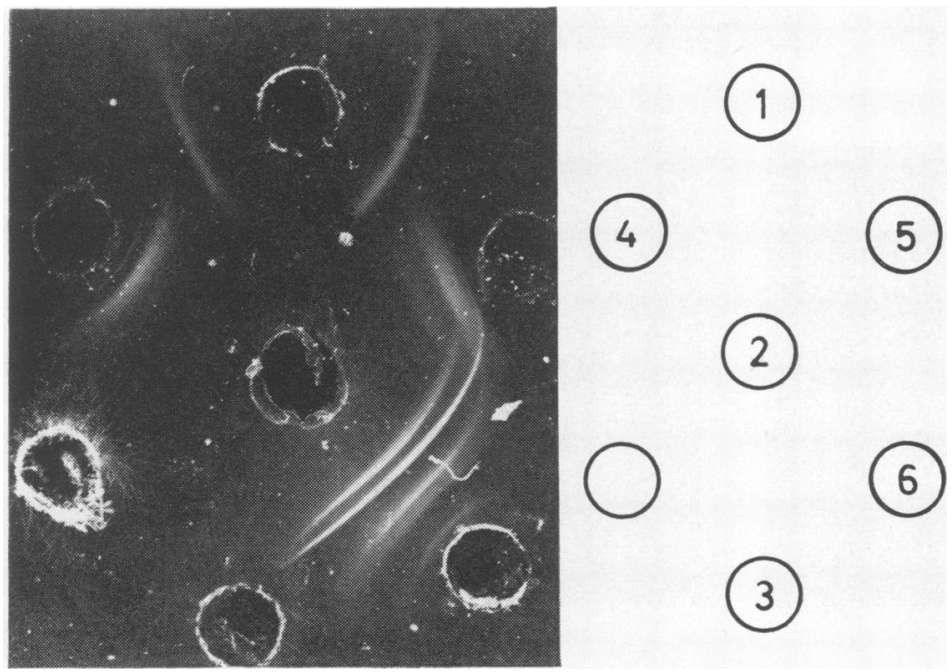

FIG. 1. Double immunodiffusion on a microplate; gastric sulphoglycoproteins of the dog and rabbit antiserum prepared against them.

$1=$ rabbit antiserum prepared against

sulphoglycoproteins of control group and absorbed with sulphoglycoproteins of aspirin group.

$2=$ rabbit antiserum prepared against sulphoglycoproteins of aspirin group. $3=$ as in 1.

4 = sulphoglycoprotein antigens of control group.

$5=$ as in 4.

$6=$ sulphoglycoprotein antigens of aspirin group.

Note the qualitative difference between the sulphoglycoproteins derived from the respective control and aspirin groups. 


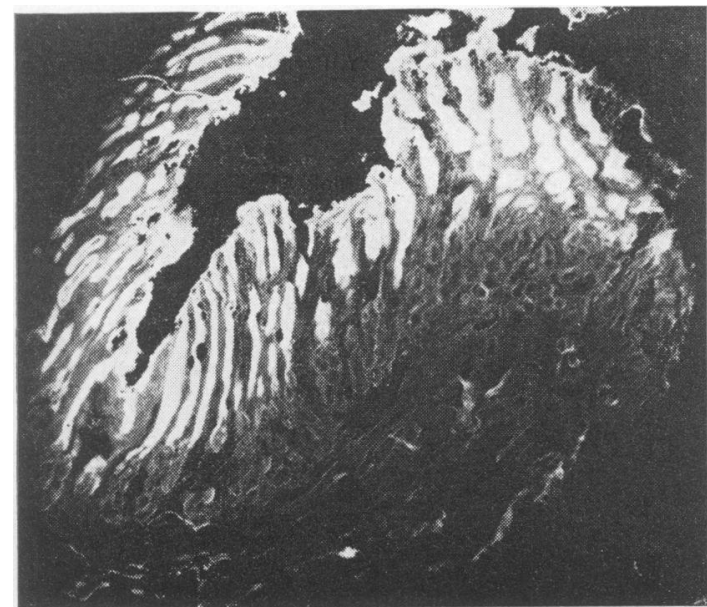

FIG. 3 .
FIG. 3. Parallel section to Fig. 2 stained with rabbit antiserum prepared against gastric sulphoglycoproteins of control group and absorbed with gastric sulphoglycoproteins of aspirin group. Deep pyloric glands are not stained.

FIG. 4. Photomicrograph of a section from the body of a stomach in control group stained as in Figure 2. Note the unstained deep glands.

FIG. 5. Parallel section to Fig. 4 stained as in Figure 3. Note the difference as compared with Fig. 4: the mucous neck cell area is not stained.

FIG. 6. Photomicrograph of a section from antrum of a stomach in aspirin group stained as in Figure 2. Diminished staining intensity as compared with Figure 2.

FIG. 7. Parallel section to Fig. 6 stained as in Figure 3. Only a trace of specific fluorescence can be seen.

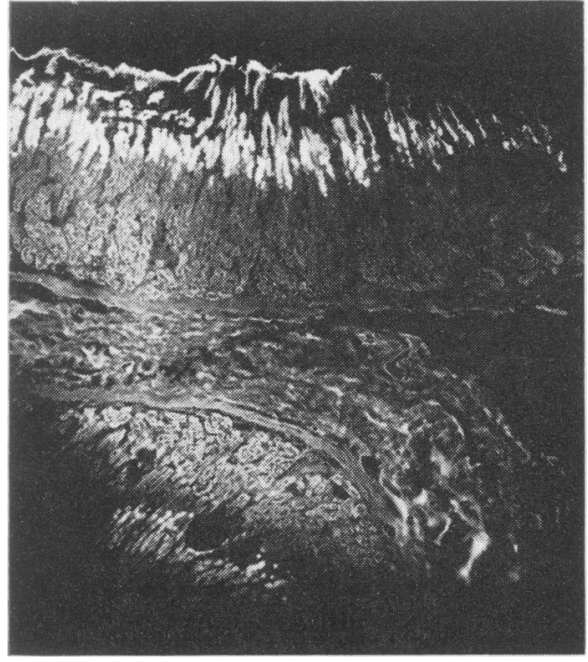

FIG. 4.

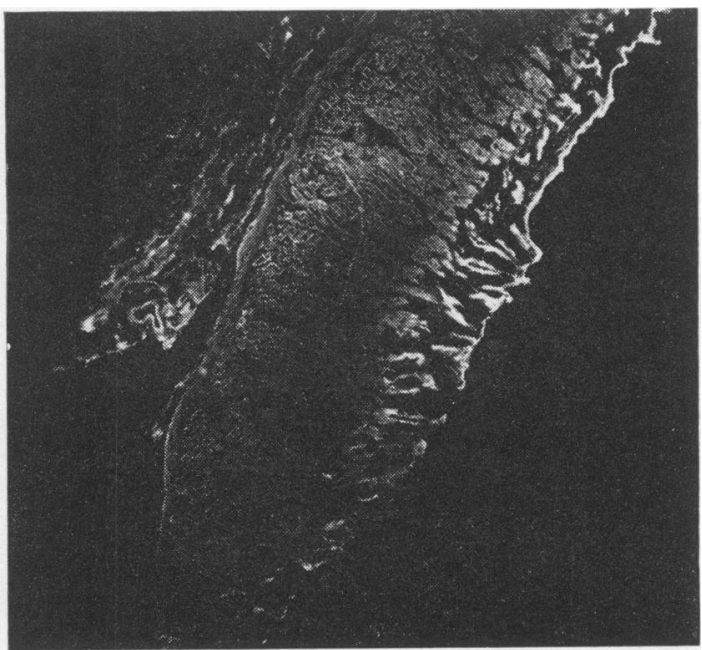

FIG. 5 .

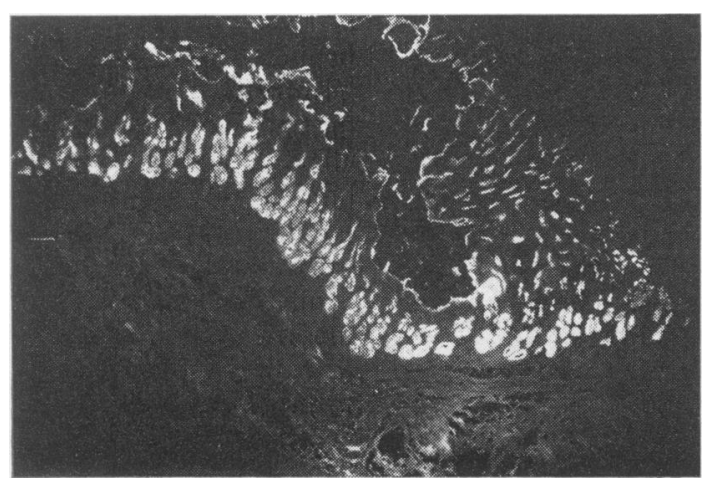

FIG. 6.

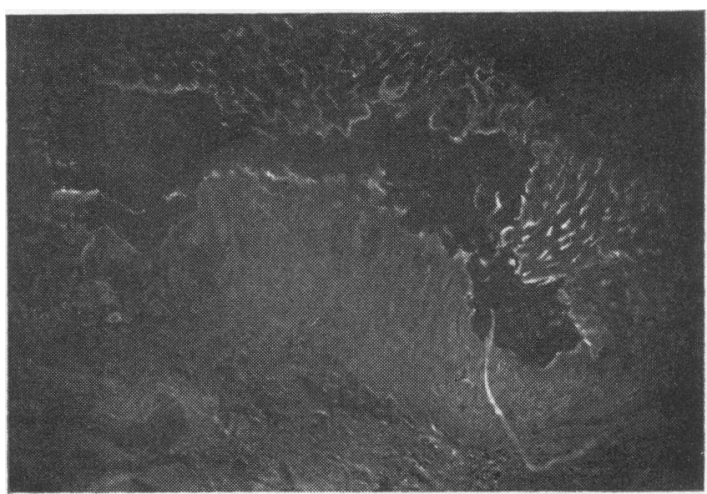

FIG. 7. 


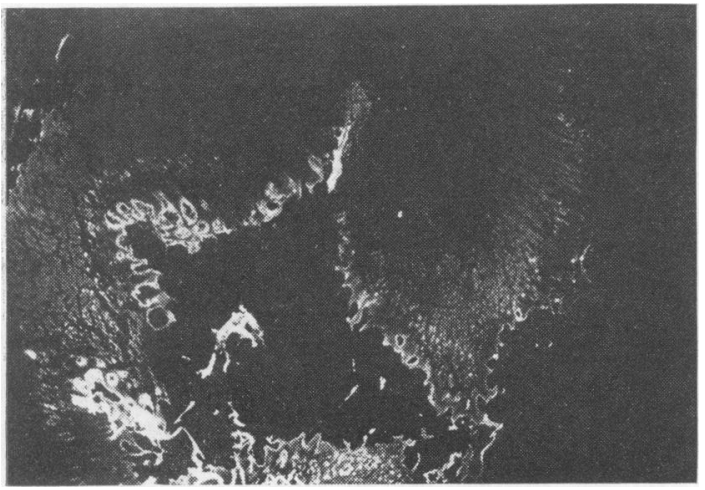

FIG. 8. Photomicrograph of a section from the body of a stomach in aspirin group stained as in Figure 2. Note the diminished specific fluorescence.

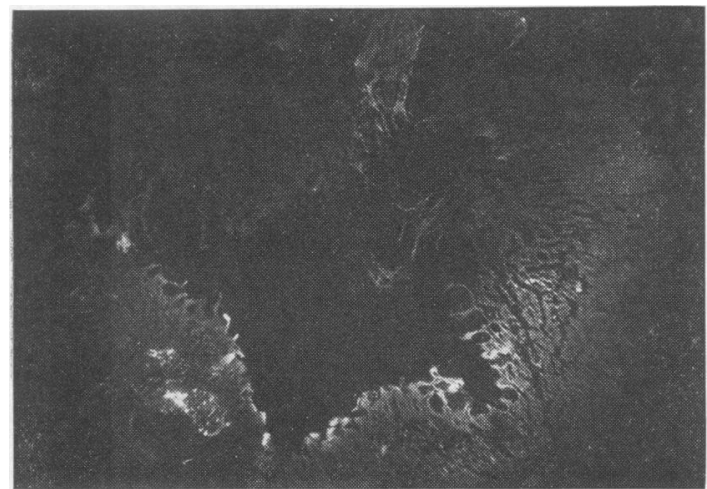

FIG. 9. Parallel section to Fig. 8 stained as in Figure 3. Only a trace of specific fluorescence can be seen.

between the control dogs. On the other hand, in the aspirin group the intensity varied appreciably from dog to dog. From the observations of qualitative differences in parallel sections stained with different immunosera (Figs. 2, 3, and 5) the following conclusions can be drawn: a normal, unstimulated dog secretes two immunologically distinguishable sulphoglycoproteins, one of which is secreted by superficial mucous cells only and the other by the same superficial cells, and also by deep pyloric glands in the antrum and by mucous neck cells in the body and fundus. Under the influence of aspirin (Figs. 6, 7, 8 , and 9) the sulphoglycoproteins secreted by superficial cells only disappear. The sulphoglycoprotein component of deep glands shows a similar tendency but to a lesser extent. When identical sections are stained with alcian blue at $p \mathrm{H} 1$, a difference between the control and aspirin-treated dogs can be seen but is not very striking. The conclusion can be drawn that the alcian blue-stained sulphomucin in aspirin dogs is mainly that of the immunological 'deep gland type'. When stained with PAS, practically no difference was obtained between the aspirin-treated and control groups. Also this favours the assumption that aspirin has a highly selective inhibitory effect on the synthesis of superficial gastric sulphoglycoprotein.

In the aspirin group there were occasional sections where the superficial epithelium was stained very weakly with alcian blue at $p \mathrm{H} 1$, one of them from a dog without any macroscopical lesions in the stomach, and but a trace of specific fluorescence in the mucosa.

\section{DISCUSSION}

It has been established earlier (Häkkinen et al, 1965; Häkkinen, 1966, 1967) that the isolation procedure used in the present study really yields sulphoglycoproteins, and another proof is that the rabbit immunosera prepared had no visible reactions with dog serum in double immunodiffusion in control experiments. The survey of neutral glycoproteins of gastric juice has been omitted in this study because of difficulties in the isolation in immunologically pure fractions.

The antibodies formed in rabbits are apparently not directed against the sulphate groups but presumably against some sugar configuration in these macromolecules, perhaps after the model in the case of antigens of blood group substances (Watkins, 1966). An assumption could be made that the neutral glycoproteins contain the same sugar antigens as the sulphated ones. If so, the specific immunofluorescence could have been caused by the presence of both kinds of substance. This possibility cannot be wholly excluded. In the case of aspirintreated mucosa the difference in histochemical stainability with alcian blue at $p \mathrm{H} 1$ (no stain) and with PAS (nearly unaltered staining), as well as the virtually total absence of specific immunofluorescence in the mucosa, strongly points to the fact that only neutral glycoproteins would be present in the mucosa and that the specificity of the immunofluorescence in this case would be connected with the sulphated macromolecules.

In any case, it seems to be ascertained that the mucous cells of gastric epithelium synthesize macromolecules provided with two kinds of antigenic determinants (or groups of determinants); one of them is formed in the superficial mucous cells only, the other also in the antral pyloric type of glands and in the mucous neck cells of the body and fundus. Especially interesting is the component which is specific for superficial cells only. The synthesis of this component seems to be easily disturbed in the 
presence of aspirin. The superficial gastric mucous cells in man secrete a specific sulphoglycoprotein (Tyrkkö, Häkkinen, and Rimpelä, 1968) which has the highly active serological specificity of a blood group substance (Häkkinen and Virtanen, 1967). It is likely that the synthesis of the glycoprotein with blood group activity would be one of the most complicated and most specialized of all gastric glycoproteins. The analogous canine compound could have a similar character.

The question of the physiological role of the gastric superficial sulphoglycoprotein can be answered in the light of the observed effect of aspirin. It has been accepted that the mucous film covering the mucosal linings protects the epithelial cells from digestive damage by pepsin and hydrochloric acid. The nature of this protection is obscure. On the other hand, it is known that certain sulphated macromolecules like gastric sulphoglycoproteins are able to form complexes with pepsin and thus inactivate it (Cayer and Ruffin, 1967; Sun, 1967). It is highly probable that in normal conditions this happens to pepsin molecules when they strike the epithelial surface. If the suitable sulphoglycoprotein is absent or insufficiently present the digestive action of pepsin may freely affect the gastric cells. The experimental conditions and methods of the present work can be applied to studies of other pharmacologically potent substances or of substances suspected of playing an aetiological role in gastric diseases.

\section{SUMMARY}

Sulphoglycoproteins present in the gastric juice of the dog have been separated in two components using immunological and histoimmunological methods. One of these components is apparently secreted only by the superficial mucous cells of the stomach, while the other component derives mainly from the deep pyloric type glands in the antral part of the stomach and from the mucous neck cell area in the corpus and cardia, and, to a lesser extent, from the superficial mucous cells.

Prolonged feeding of aspirin had a selective inhibitory effect on the secretion and presumably on the synthesis of gastric sulphoglycoproteins in the dog. Immunological, histoimmunological, and histochemical data uniformly point to the fact that the sulphoglycoprotein component specific to superficial mucous cells is mainly affected. This specific sulphoglycoprotein component in the dog might be analogous to the human gastric sulphoglycoprotein also synthesized by superficial gastric mucous cells. The physiological importance of superficial gastric sulphoglycoprotein as an inhibitory factor for pepsin digestion is discussed.

This study has been supported by grants from the Damon Runyon Memorial Fund DRG-977 and the Finnish National Medical Research Council.

\section{REFERENCES}

Cayer, D., and Ruffin, J. M. (1967). Effect of depepsen in the treatment of peptic ulcer. Ann. N.Y. Acad. Sci., 140, 744-746.

Davis, B. J. (1964). Disc electrophoresis. II. Method and application to human serum proteins. Ibid., 121, 404-427.

Grossman, M. I., Matsumoto, K. K., and Lichter, R. J. (1961). Fecal blood loss produced by oral and intravenous administration of various salicylates. Gastroenterology, 40, 383-388.

Häkkinen, I. P. T. (1966). An immunochemical method for detecting carcinomatous secretion from human gastric juice. Scand. J. Gastroent., 1, 28-32.

- (1967). Differentiation of antigenic gastric cancer sulphopolysaccharides from metaplastic 'Intestinal' type sulphopolysaccharides. Ibid., 2, 39-43.

- Hartiala, K., and Terho, T. (1965). The fractionation and charactelization of the acid polysaccharides in human gastric juice. Acta chem. scand., 19, 797-799.

-, Grönroos, J., and Kunnas, R. (1967). Sulphoglycoproteins in normal, metaplastic and cancerous gastric mucosa as detected by immunofluorescence technique. Ann. Med. exp. Fenn., 45, 206-212.

- , and Virtanen, S. (1967). The blood group activity of human gastric sulphoglycoproteins in patients with gastric cancer and normal controls. Clin. exp. Immunol., 2, 669-675.

Robbins, P. W., Bray, D., Dankert, M., and Wright, A. (1967). Direction of chain growth in polysaccharide synthesis. Science, 158, 1536-1542.

Roth, J. L. A., Valdes-Dapena, A., Pieses, P., and Buchman, E. (1963). Topical action of salicylates in gastrointestinal erosion and hemorrhage. Gastroenterology, 44, 146-158.

Spicer, S. S., and Sun, D. C. H. (1967). Carbohydrate histochemistry of gastric epithelial secretions in dog. Ann. N.Y. Acad. Sci., 140, 762-783.

Sun, D. C. H. (1967). Effect of a synthetic sulfated polysaccharide (SN-263) on gastric peptic activity in humans. Ibid., 140, 747753.

Tyrkkö, J., Häkkinen, I., and Rimpelä, U. (1968). On the histochemical demonstration of sulphomucins in human and canine gastric epithelium. Brit. J. exp. Path. 4, 371-374.

Watkins, W. M. (1966). In Glycoproteins: Their Composition, Structure and Function, p. 510. Elsevier, Amsterdam and Barking, Essex. Edited by A. Gottschalk.

Whitehouse, M. W. (1964). Biochemical properties of anti-inflammatory drugs. III. Uncoupling of oxidative phosphorylation in a connective tissue (cartilage) and liver mitochondria by salicylate analogues:relationship of structure to activity. Biochem. Pharmacol., 13, 319-336.

- and Boström, H. (1962). The effect of some anti-inflammatory (anti-rheumatic) drugs on the metabolism of conective tissues. Ibid., 11, 1175-1201. 